\title{
Oxidation of Copper Nanoparticles Protected with Different Coatings and Stored under Ambient Conditions
}

\author{
Noemi Jardón-Maximino, ${ }^{1}$ Marissa Pérez-Alvarez $\mathbb{D}^{2},{ }^{2}$ Rubén Sierra-Ávila ${ }^{\circledR}{ }^{1}$ \\ Carlos Alberto Ávila-Orta ${ }^{(D)},{ }^{1}$ Enrique Jiménez-Regalado ${ }^{(D},{ }^{1}$ Angélica Mara Bello $\left(\mathbb{D},{ }^{3}\right.$ \\ Pablo González-Morones $\mathbb{D}^{1},{ }^{1}$ and Gregorio Cadenas-Pliego ${ }^{1}{ }^{1}$ \\ ${ }^{1}$ Centro de Investigación en Química Aplicada, 25294 Saltillo, COAH, Mexico \\ ${ }^{2}$ CONACyT Research Fellow-Instituto Mexicano del Petróleo, 07730 Ciudad de México, Mexico \\ ${ }^{3}$ Tecnológico Nacional de México, Instituto Tecnológico de Orizaba, 94320 Orizaba, VER, Mexico \\ Correspondence should be addressed to Marissa Pérez-Alvarez; mperezal@conacyt.mx \\ and Gregorio Cadenas-Pliego; gregorio.cadenas@ciqa.edu.mx
}

Received 8 June 2018; Revised 6 July 2018; Accepted 22 July 2018; Published 19 September 2018

Academic Editor: Xuping Sun

Copyright (c) 2018 Noemi Jardón-Maximino et al. This is an open access article distributed under the Creative Commons Attribution License, which permits unrestricted use, distribution, and reproduction in any medium, provided the original work is properly cited.

\begin{abstract}
The synthesis of copper nanoparticles ( $\mathrm{Cu}$ NPs) stable to chemical oxidation has attracted much attention due to their novel application possibilities. The physicochemical properties of $\mathrm{Cu}$ NPs define their utilization in diverse fields, including biological applications, solar cells, high conductivity inks for printed electronics, and other specific applications. In this study we present the synthesis of oxide-free $\mathrm{Cu}$ NPs by wet chemistry routes and their chemical oxidation during the storage exposed to ambient conditions. The $\mathrm{Cu}$ NPs synthesized in PAAm and AAm/glycerol presence exhibit no oxidation during synthesis and atmospheric conditions storage for 60 and 37 months, respectively. The obtained results compared with those coming from oxidation of partially passivated commercial Cu NPs at $99.8 \%$ purity, are presented. The role of the $\mathrm{Cu}$ NPs coating is critical to avoid oxidation, and it can be an important feature to achieve good dispersion in different polymers. The Cu NPs oxidation was studied by XRD, TGA, TEM, FTIR, and HR-TEM.
\end{abstract}

\section{Introduction}

In the last decade, studies related to synthesis and application of $\mathrm{Cu}$ NPs has been of great interest from the academic and industrial point of view, due to its large potential for the replacement of expensive metals like silver and gold nanoparticles in the production of antibacterial and conductive materials $[1,2]$. Research regarding the use of $\mathrm{Cu}$-based materials continues to grow in many diverse fields, for example, in 2018, its use in membranes for water purification [3], agriculture [4, 5], electronics $[6,7]$, catalysis [8-11], and so on, was reported.

Recently, our research group reported the synthesis of $\mathrm{Cu}$ NPs by chemical reduction in aqueous solution and thermal decomposition of copper precursors [12-14].
Several synthetic methods for the preparation of copper nanoparticles have been developed including thermal and sonochemical reduction [15], metal vapor synthesis (MVS) [16], electron beam irradiation [17], microwave irradiation [18], reverse micelles [19], pulsed laser ablation [20], chemical reduction in aqueous solution [21], and polyol process [22]. Most of the preparation methods have several factors that limit their use in the synthesis of metal particles, among these synthetic strategies, the wet chemical reduction is one of the most convenient methods because it has unique advantages over the other methods due to its versatility, the water is inexpensive, and has the ability to solubilize a wide variety of metallic salts, wherein the nanoparticles obtained by this method have well-defined shapes and sizes [23]. However, only in few cases $\mathrm{Cu}$ NPs are exclusively obtained; typically, 
they are obtained along with copper oxide II $(\mathrm{CuO})$ and copper oxide $\mathrm{I}\left(\mathrm{Cu}_{2} \mathrm{O}\right)$ impurities due to relatively $\mathrm{Cu}^{0} / \mathrm{Cu}^{2+}$ low reduction potential.

Numerous methods for $\mathrm{Cu}$ NP synthesis have been reported using chemical reduction in aqueous solution. To prevent the copper nanoparticle oxidation, the use of stabilizers or agents such as water-soluble coating polymers, surfactants, or coordination ligands that have functional groups with the ability to form complexes with copper ions present in the medium is required [23]. Using hydrazine hydrate $\left(\mathrm{N}_{2} \mathrm{H}_{4} \cdot \mathrm{H}_{2} \mathrm{O}\right)$ as reducing agent aids the $\mathrm{Cu}$ NP protection due to the $\mathrm{N}_{2}$ production during the oxidation process by eliminating the oxygen present in the reaction media.

$\mathrm{Cu} \mathrm{NP}$ synthesis in polyallylamine (PAAm) presence using $\mathrm{N}_{2} \mathrm{H}_{4} \cdot \mathrm{H}_{2} \mathrm{O}$ as reducing agent for $\mathrm{CuSO}_{4} \cdot 5 \mathrm{H}_{2} \mathrm{O}$ yields colloidal copper nanoparticles (oxide free) exhibiting large surface-enhanced Raman scattering (SERS) signals. Cu NPs are obtained without caring about argon or nitrogen environment inert atmosphere, the PAAm act as surface protectors, having the function of controlling size, size distribution, and shape of particles, and also preventing agglomeration thereof [24].

Using soluble polymers or surfactants as capping agents to prepare $\mathrm{Cu}$ nanoparticles in aqueous solutions is attractive because organic solvents are avoided and the corresponding pollutants are absent. However, until now, only few works have been done since $\mathrm{Cu}$ is easily oxidized, making it difficult to handle.

Other strategies to protect copper nanoparticles against chemical oxidation during preparation and storage is to perform the synthesis in a nonaqueous medium using a low concentration of metal precursor and typically they are made under inert atmospheres of $\mathrm{Ar}$ or $\mathrm{N}_{2}$ [25]. Other methods synthesize $\mathrm{Cu}$ NPs in the absence of water and high temperature producing carbon-coated NPs. One example is the copper II carbonate reduction by glucose in solid state and $350^{\circ} \mathrm{C}$ temperature to produce $\mathrm{Cu}$ NPs with an amorphous carbon coating to avoid oxidation [26].

The $\mathrm{Cu}$ NP synthesis using a flame spray reactor under nitrogen atmosphere yields the continuous production of $\mathrm{Cu}$ NPs covered with approximately $1 \mathrm{~nm}$ carbon coating, protecting them from air-caused oxidation [27]. The Cu NP synthesis by thermal decomposition of copper (II) acetate in diphenyl ether in the presence of different capping ligand produces organically coated $\mathrm{Cu} \mathrm{NP}$ air stable, after exposing the sample to air for $24 \mathrm{~h} \mathrm{[28].}$

The oxidation of different $\mathrm{Cu}$ NPs has been reported in the literature; however, these studies are incomplete, and learning about the initial oxidation and oxidation progress through time in different environmental conditions is needed. The value of this knowledge is to learn the oxidation degree of the Cu NPs after storage and to know the role of the coating in the chemical stability. In this study, we analyze the $\mathrm{Cu}$ NP oxidation synthesized by chemical reduction method in aqueous solution, having the presence of organic ligands and using $\mathrm{N}_{2} \mathrm{H}_{4} \cdot \mathrm{H}_{2} \mathrm{O}$ as reducing agent. Furthermore, the oxidation of partially passivated commercial $\mathrm{Cu}$ NPs with $99.8 \%$ purity is also studied.
The significance of this study lies in the general lack of characterization of $\mathrm{Cu}$ NPs after storage and later they are used without knowledge of the exact oxidation degree at the time of use. This common practice can produce altered and or diverse results in the $\mathrm{Cu}$ NP performance.

\section{Experimental}

2.1. Materials and Reagents. Copper (II) sulfate pentahydrate, hydrazine hydrate solution (50-60\%), poly(allylamine) solution (PAAm) ( $\mathrm{Mw} \sim 17,000,20 \mathrm{wt} \%$ aqueous solution), allylamine (AAm) (98\%), and sodium hydroxide (98.8\%) were obtained from Sigma-Aldrich and were used without previous purification. Cu NPs partially passivated $(25 \mathrm{~nm}$, 99.8\%) was obtained from SkySpring Nanomaterials Inc.

2.2. Synthesis of Copper Nanoparticles. The synthesis of copper nanoparticles was performed using wet chemical reduction.

2.2.1. Method 1. In a flat bottom flask, $\mathrm{CuSO}_{4} \cdot 5 \mathrm{H}_{2} \mathrm{O}$ was added and dissolved in distilled water under magnetic stirring for 10 minutes at $60^{\circ} \mathrm{C}$. PAAm was dissolved in distilled water and later was added in a dropwise manner and stirred for 10 minutes. Subsequently, a solution of $0.5 \mathrm{M} \mathrm{NaOH}$ was added dropwise; the reaction mixture was allowed to react for 30 minutes followed by the addition of hydrazine and constant stirring was maintained at $60^{\circ} \mathrm{C}$. The nanoparticles were recovered by centrifugation at $15,000 \mathrm{rpm}$ and $25^{\circ} \mathrm{C}$, purification was realized after washing twice with distilled water and ethanol and finally dried at $60^{\circ} \mathrm{C}$ for 2 hours; the NPs were obtained as black powder y and labeled as Cu NPs $1[12,14]$.

2.2.2. Method 2. $\mathrm{CuSO}_{4} \cdot 5 \mathrm{H}_{2} \mathrm{O}(20 \mathrm{~g})$ was placed in a glass reactor and $200 \mathrm{ml}$ of deionized water was added. The temperature was adjusted to $60^{\circ} \mathrm{C}$ and the mixture was stirred at $600 \mathrm{rpm}$. Glycerine $(40 \mathrm{ml})$ was added using an addition funnel and after 3 min stirring; allylamine $(78 \mathrm{ml})$ was immediately added followed by $30 \mathrm{~min}$ stirring. Finally, hydrazine $(80 \mathrm{ml})$ was added dropwise, keeping temperature and stirring for $2 \mathrm{~h}$ and $30 \mathrm{~min}$ more. After this treatment, $\mathrm{Cu}$ NPs were purified following the previously described procedure. The $\mathrm{Cu}$ NPs are obtained as a red brown color powder and labeled as CuNPs 2.

2.2.3. Cu NP Storage. The Cu NPs obtained by the methods 1 and 2 were stored in polyethylene bags and were exposed to the air and light during the complete study. After opening, the commercial $\mathrm{Cu}$ NPs were stored in a Schlenk tube and kept under argon atmosphere. One small sample was stored in a glass vial and exposed to air and light conditions during the complete study.

2.3. Characterization Techniques. X-ray diffraction (XRD) technique was employed in order to know the oxidation state and chemical stability of the synthesized copper nanoparticles on a Siemens D-5000 diffractometer with a scanning interval in the $2 \theta$ range of 30 to $80^{\circ}$, with a scan speed of $0.02^{\circ} / \mathrm{s}$, the radiation employed was copper $\mathrm{K} \alpha$ with a 


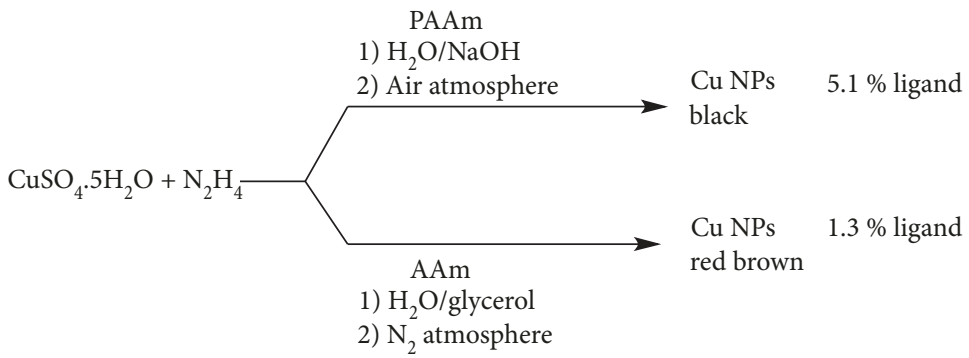

Figure 1: Synthesis of Cu NPs with different ligands.

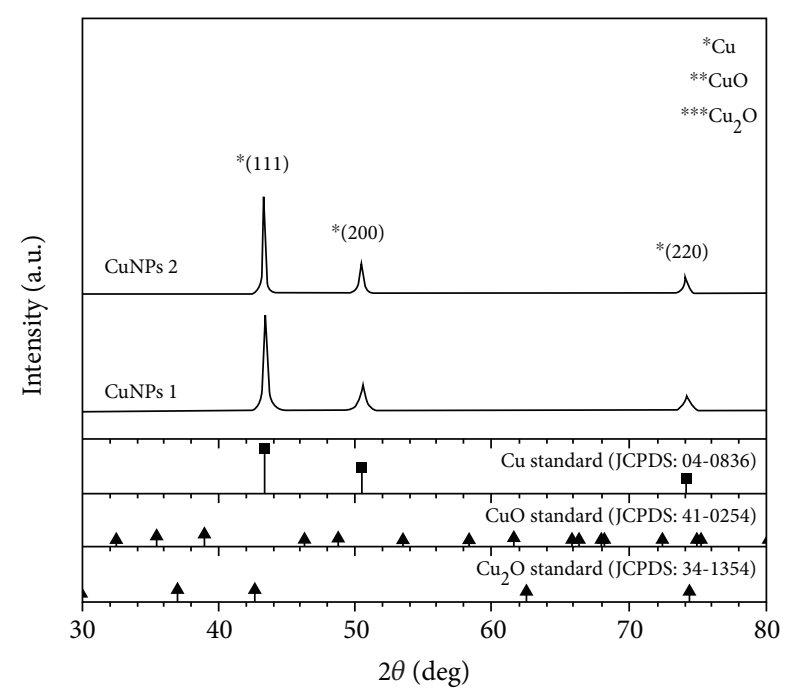

FIGURE 2: XRD diffractograms of copper nanoparticles synthesized by chemical reduction method.

wavelength of $1.54056 \AA$, values of $25 \mathrm{~mA}$ and $35 \mathrm{kV}$ were used for intensity and voltage, respectively. The average particle diameter was determined from the XRD diffractograms of each sample based on the Debye-Scherrer equation, taking the most intense peak for calculation [29, 30]. Transmission electron microscopy (TEM) was used in order to observe the morphology and particle size distribution of the copper samples on a FEI Titan high-resolution electron microscope operating at $300 \mathrm{kV}$.

Thermogravimetric analysis (TGA) was employed to analyze the thermal behavior of $\mathrm{Cu}$ particles and the thermal stability of coating using a Du Pont Instruments 951 analyzer. Operating conditions were a heating rate of $10^{\circ} \mathrm{C} / \mathrm{min}$., with a gas flow of $50 \mathrm{ml} / \mathrm{min}$. The runs of the samples were carried out from $30^{\circ} \mathrm{C}$ to $600^{\circ} \mathrm{C}$ in $\mathrm{N}_{2}$ atmosphere. Once $600^{\circ} \mathrm{C}$ was reached, $\mathrm{N}_{2}$ atmosphere was changed for $\mathrm{O}_{2}$.

Fourier transform infrared spectroscopy (FTIR) spectra of samples were recorded in a FTIR Spectrometer GX, PerkinElmer.

\section{Results and Discussion}

3.1. Cu NP Synthesis and Characterization. The Cu NP synthesis obtained by the chemical reduction method in aqueous media (methods 1 and 2) produces exclusively $\mathrm{Cu}$ NPs. When PAAm is used, the nanoparticles are obtained as black color powder with $5.1 \%$ ligand content and $76 \%$ yield, while using AAm and glycerol produces a red brown color, $1.3 \%$ ligand content and $86 \%$ yield. The $\mathrm{Cu}$ NPs were handled in air atmosphere since separation and purification processes. Finally, the products were stored in polyethylene bags in normal environmental conditions. Figure 1 shows the scheme of synthesis for the $\mathrm{Cu}$ NPs.

The Cu NPs obtained by methods 1 and 2 were characterized immediately after synthesis by XRD, TGA, and TEM techniques. The X-ray diffraction patterns for both $\mathrm{Cu}$ NPs synthesized methods exhibit three reflections located at $2 \theta=43.4,50.5$, and $74.0^{\circ}$, attributed to the (111), (200), and (220) crystal planes, respectively, belonging to pure copper with face-centered cubic symmetry (FCC) $[31,32]$ and corresponding to the diffraction pattern of metallic copper (JCPDS No. 04-0836) [33], as shown at the bottom of Figure 2.

The XRD diffractograms of the particles obtained show no reflections that correspond to $\mathrm{CuO}$ NPs. A comparison with the standards shown at the bottom of the graph readily identifies each type of NPs (JCPDS.0254).

The X-ray characterization showed the protection capacity of the nitrogenated ligands during the synthesis and further handling to the $\mathrm{Cu}$ NPs avoiding oxidation when exposed to an open environment. Experimental data support several studies showing the protection feature of the presence of nitrogenated ligands (polymers and/or organic molecules); TGA characterization results provided more information on the thermal stability of the used ligands.

The $\mathrm{Cu}$ NPs obtained by methods 1 and 2 in PAAm and allylamine presence, respectively, were also characterized by TGA (Figure 3). The Cu NP thermogram obtained with the polymeric PAAm exhibited the highest mass loss in the $200^{\circ} \mathrm{C}$ and $494^{\circ} \mathrm{C}$ temperature range; the maximum degradation temperature of the polymeric covering was found in $440^{\circ} \mathrm{C}$. The described thermic behavior is typical for polymers with thermic decomposition under $500^{\circ} \mathrm{C}$ temperature. The PAAm covering avoids oxidation of the Cu NPs up to $600^{\circ} \mathrm{C}$; after this temperature, the nitrogen environment is exchanged to oxygen and a weight gain is observed as a result of the metallic copper oxidation. The thermogram of the synthesized $\mathrm{Cu}$ NPs in presence of the AAm ligand (Figure 3) shows a weight loss from $50^{\circ} \mathrm{C}$ until $260^{\circ} \mathrm{C}$ temperature, the maximum degradation temperature of the coating was found 


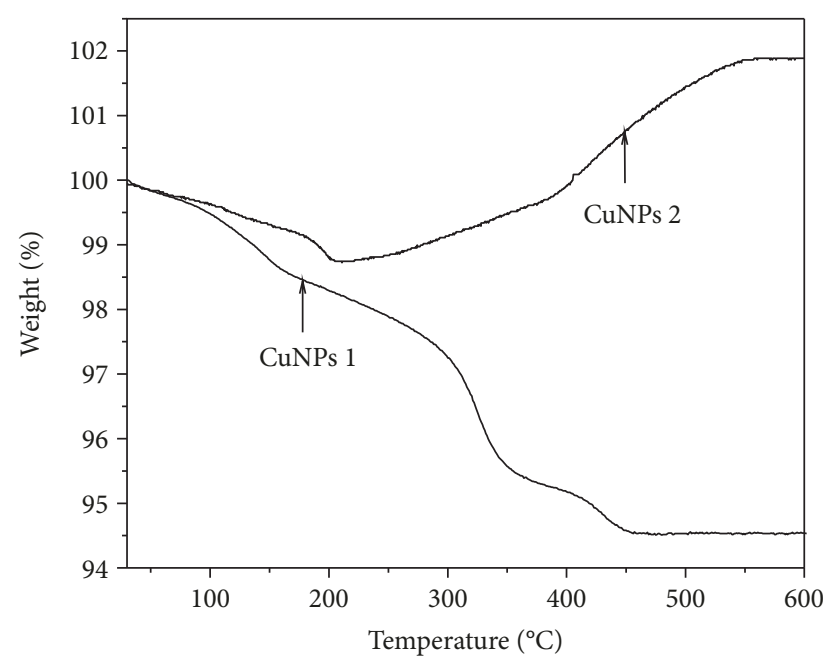

FIGURE 3: TGA thermograms of copper nanoparticles synthesized through chemical reduction with PAAm and AAm.

at $210^{\circ} \mathrm{C}$, the value is lower compared to that observed for the PAAm coating. The temperature range for losing the Cu NPs covering suggests that this covering can be made of molecules used during the synthesis ( $\mathrm{AAm}, \mathrm{H}_{2} \mathrm{O}$ y glycerol), this result shows the lack of polymerization for AAm as previously reported in basic media [14], and despite using a large amount of AAm, only a small amount is incorporated into the $\mathrm{Cu}$ NPs 2.

In studies done previously, ${ }^{1} \mathrm{H}$ NMR, FTIR, and GS-Ms techniques demonstrate that $\mathrm{CuSO}_{4} \cdot 5 \mathrm{H}_{2} \mathrm{O}$ reduction in presence of AAm using $\mathrm{N}_{2} \mathrm{H}_{4} / \mathrm{NaOH}$ yields AAm polimerization and PAAm generation. New characterization using permeation chromatography (GPC) results show high molecular weight PAAM. These distinctive results suggest that basic media is an essential condition to favour AAm polymerization.

The PAAm-coated NP (CuNPs 1) characterization was performed by transmission electronic microscopy (TEM). The micrograph shows isolated particles with sizes between 20 and $30 \mathrm{~nm}$ and presenting a hemispheric morphology (Figure $4(\mathrm{a})$ ). Also, the presence of agglomerates with diameters between 50 and $200 \mathrm{~nm}$ can be noticed, which are formed by subparticles with approximate sizes in the range of 18 to $50 \mathrm{~nm}$. The black arrow points out the enlargement of a polycrystalline aggregate of nanoparticles whose surface is wrapped by a thin layer of PAAm with a thickness of about $5 \mathrm{~nm}$, acting as a surface coating.

The Cu NP micrographs obtained with AAm (CuNPs 2) showed particles with a well-defined morphology, sphere shaped, and particle size between 5 and $20 \mathrm{~nm}$ (Figure 4(b)). TEM analyses suggest that PAAm ligand favored the particle agglomeration (Figure 4(a)). This theory is self-sustaining if the amino groups present in the ligand are distributed along the polymer chain and are localized in a single region while the small molecules such as AAm and/or glycerol are totally free.

The Cu NP energy dispersive X-ray spectroscopy (EDS) analysis showed the presence of $\mathrm{Cu}$ as the main element and traces of carbon and oxygen were also found, nitrogen was not detected in any of the analyzed samples.

In order to obtain further evidence of the coating, the CuNPs were analyzed by high-resolution transmission electron microscopy (HR-TEM). By using this technique, it successfully detected isolated CuNPs 1 and CuNPs 2 exhibiting spherical morphology with a diameter of $29.3 \mathrm{~nm}$ and $22.6 \mathrm{~nm}$, respectively (Figures 5(a) and 5(b)), HR-TEM image shows the coating the copper nanoparticles having a thickness less than $1 \mathrm{~nm}$ in both cases. The color of the coating is lighter in comparison to the copper nanoparticles one. The HR-TEM images of CuNPs 2 clearly exhibit the $\mathrm{Cu}$ lattice planes (Figure 5(b)); this characteristic is not observed in the CuNPs 1 (Figure 5(a)) because they show a greater amount of coating with respect to CuNPs 2 (5.1\% versus $1.3 \%)$.

Analysis of CuNPs 1 and 2 by FTIR provided evidence of the presence of the coating on the NPs. The spectrum CuNPs1 presented three intense bands located at $983 \mathrm{~cm}^{-1}$, $1449 \mathrm{~cm}^{-1}$, and $1601 \mathrm{~cm}^{-1}$; these values are very similar to those presented by commercial PAAm [14]. The FTIR spectrum corresponding to CuNPs 2 presented bands located at $613 \mathrm{~cm}^{-1}, 1108 \mathrm{~cm}^{-1}, 2922 \mathrm{~cm}^{-1}$, and $3451 \mathrm{~cm}^{-1}$; these bands are characteristic of glycerol presence [34]. An intense band was also detected in $1620 \mathrm{~cm}^{-1}$ which could correspond to $\mathrm{H}_{2} \mathrm{O}$ molecules that were not completely removed during the drying of the sample; the FTIR spectrum showed no clear evidence of the presence of AAm and PAAm.

3.2. Air-Stored Copper Nanoparticle Characterization. The commercial $\mathrm{Cu}$ NPs used in this study are sold in special packages to avoid air and light exposure; furthermore, they are partially passivated and possess $99.8 \%$ purity, this means only $0.2 \%$ of other elements can coat this kind of particles.

The commercial $\mathrm{Cu}$ NPs are immediately oxidized once exposed to air, and literature reports indicating traces of oxidized cupper content are commonly found. In this study, the commercial $\mathrm{Cu}$ NPs were exposed to air for 1 hour and later analyzed by X-ray diffraction. The diffraction pattern exhibited the typical reflections for metallic copper at $2 \theta=43.4$, 50.5 , and $74.0^{\circ}$, attributed to the (111), (200), and (220) crystal planes, respectively (Figure 6). Furthermore, one small signal was detected indicating the beginning of the oxidative process $\left(2 \theta=36.9^{\circ}\right)$. The EDS analysis detected copper as the main element and carbon and oxygen traces. The presence of oxygen confirms the partial oxidation of the metallic copper. The evidence of commercial Cu NP partial oxidation has been reported previously $[35,36]$.

The commercial $\mathrm{Cu}$ NPs stored in a glass vial without inert atmosphere were analyzed after some time. The diffraction patterns indicate the ineffectiveness of the coating to avoid the oxidation in this type of particles. After one month of storage, the diffraction pattern obtained show that the oxidation is stopped; this fact suggests a $\mathrm{CuO}$ coat formed on the particle surface, protecting the metallic copper nucleus. After 11 months of storage, the diffraction pattern showed $\mathrm{CuO}$ as the main component; low intensity peaks corresponding to $\mathrm{Cu}$ NPs were also observed. After 20 months of storage, it 


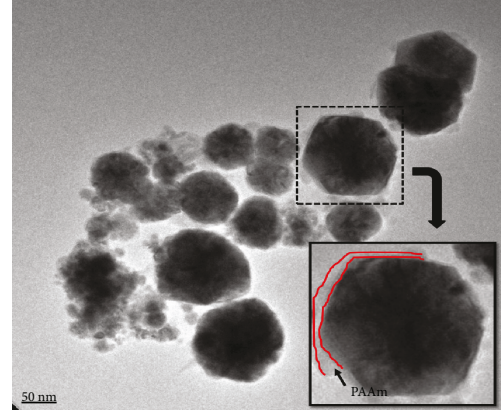

(a)

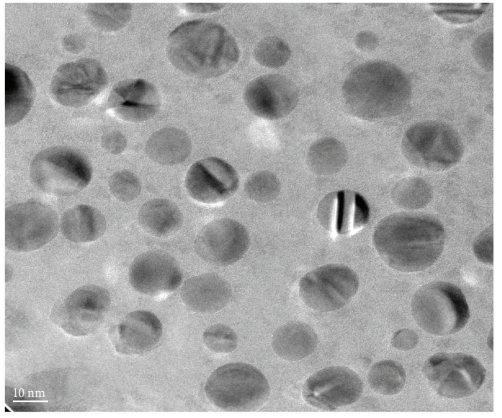

(b)

FIGURE 4: TEM micrographs of copper nanoparticles synthesized through chemical reduction.

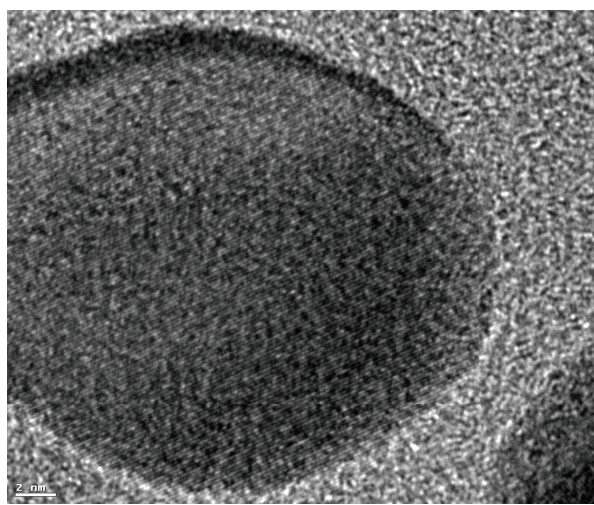

(a)

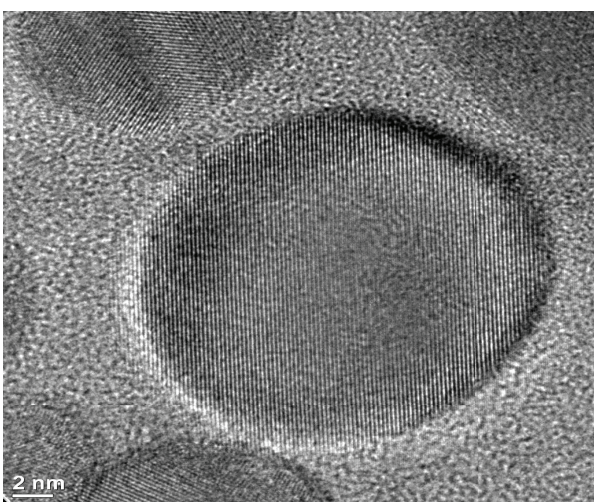

(b)

FIGURE 5: HR-TEM micrographs of copper nanoparticles synthesized through chemical reduction.

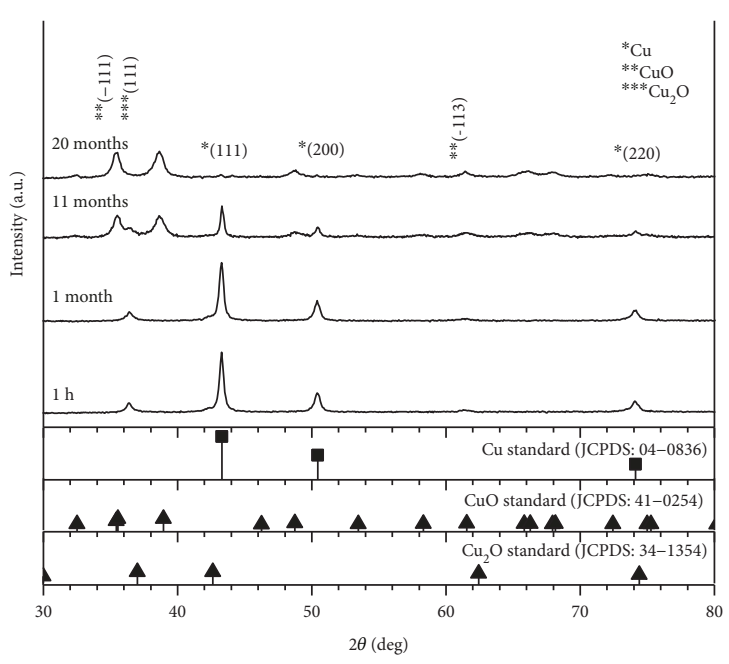

FiguRE 6: XRD diffractograms of commercial Cu NPs.

is extremely difficult to detect the Cu NP peaks, due to the oxidized copper present as a main component (Figure 6).

The TEM analysis of the commercial $\mathrm{Cu}$ NPs containing $\mathrm{CuO}$ as a main component (20 months) exhibited micrographs with a large amount of agglomerates where the spherical morphology of the original $\mathrm{Cu}$ NPs cannot be clearly observed (Figure 7).

The EDS analysis also shows a clear information supporting the transformation of $\mathrm{Cu} \mathrm{NPs}$ to $\mathrm{CuO}$; the spectrum from a recently open sample and the other after 11 months of storage show significant increase of the peak corresponding to the oxygen atom.

The $\mathrm{Cu}$ NPs synthesized in PAAm and AAm presence were also stored in similar conditions and X-ray analyzed after fixed time. The diffraction patterns of the Cu NPs synthesized in presence of PAAm, after 60 months of storage under atmospheric conditions, do not present the typical peaks belonging to $\mathrm{CuO}$ and $\mathrm{Cu}_{2} \mathrm{O}$, suggesting that the polymeric PAAm ligand coating the NP surface prevents the NP oxidation (Figure 8(a)). Similar behavior was observed with the $\mathrm{Cu}$ NPs obtained in AAm presence; in this case, the analysis was done at 37 months of storage and evidence of strong oxidation was not detected (Figure 8(b)).

The Cu NPs synthesized in PAAm and AAm presence exhibited higher stability in comparison with commercial $\mathrm{Cu}$ NPs, this suggest coordination of the nitrogen atoms to the metallic copper atom thus, avoiding the environmentally available oxygen atoms and water molecules attacking the copper. The lack of functional groups in commercial $\mathrm{Cu}$ NPs promotes the oxidation of these particles. 


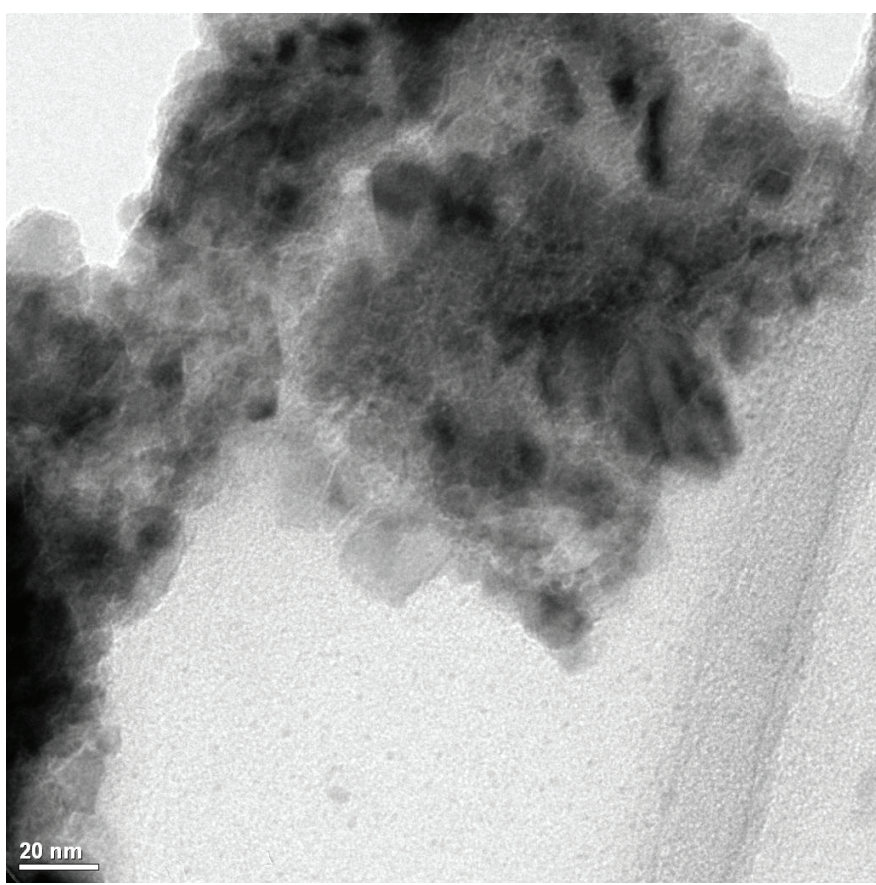

Figure 7: TEM micrograph of commercial Cu NPs.

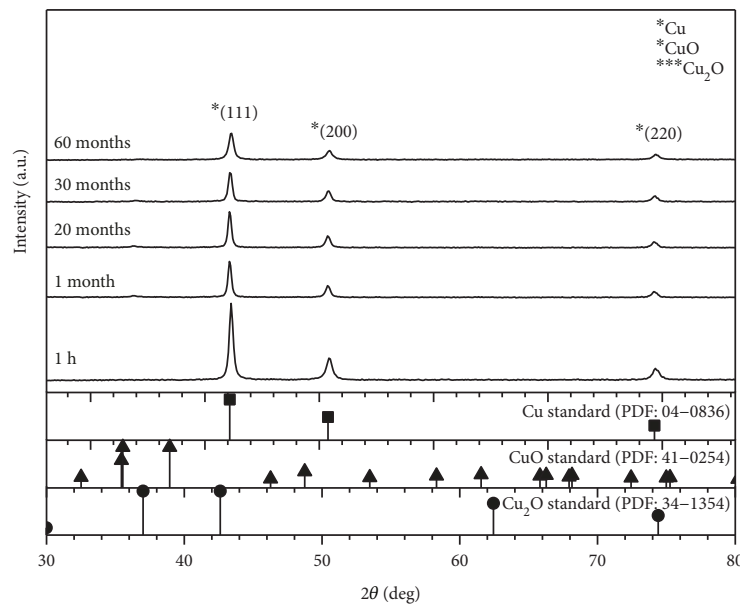

(a)

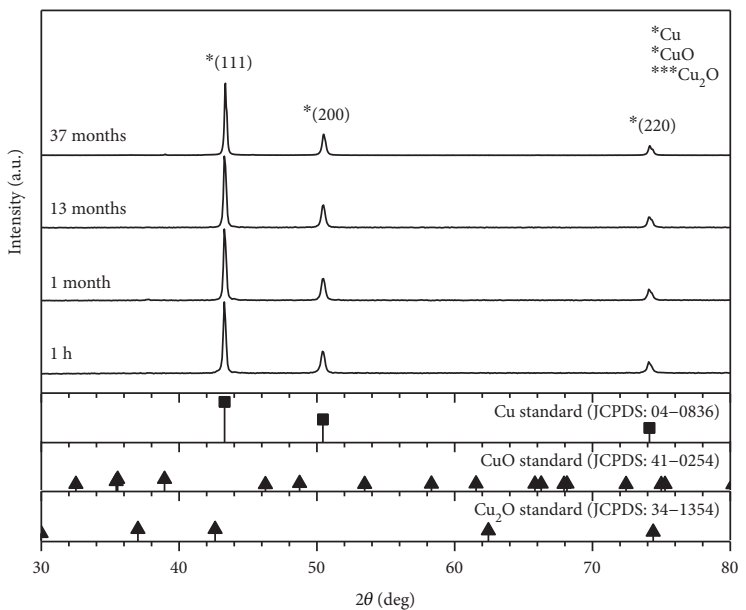

(b)

FIgURE 8: XRD diffractograms of $\mathrm{Cu}$ NPs synthesized by chemical reduction method.

In order to verify the role played by the amino groups $\left(-\mathrm{NH}_{2}\right)$ in the protection of the metallic copper, commercial CuNPs were functionalized using PAAm in aqueous solution. Unfortunately, it was not possible to perform the functionalization because commercial CuNPs are oxidized immediately upon contact with the aqueous solution; this result suggests that the surface protection of the CuNPs must be carried out in situ, that is to say, during the $\mathrm{Cu}^{2+}$ reduction.

The stability of $\mathrm{Cu}$ NPs against oxidation can play an important role in several applications and should be especially important in the antimicrobial activity because metallic $\mathrm{Cu}$ NPs are more bioreactive compared to copper oxide NPs [37].

\section{Conclusions}

The synthesis of $\mathrm{Cu}$ NPs using the chemical reduction method in aqueous phase using hydrazine as reducing agent and in presence of organic ligands produces $\mathrm{Cu}$ NPs that remain stable when exposed to air which are able to be stored in normal environmental conditions. To our knowledge, there are no reports demonstrating that $\mathrm{Cu}$ NPs can be stored 3-5 years under environmental conditions without suffering oxidation. This significant feature can play an important role in those applications that require $\mathrm{Cu}$ NPs oxide free, such as electrical current conducting inks. 


\section{Data Availability}

The data used to support the findings of this study are available from the corresponding author upon request.

\section{Conflicts of Interest}

The authors declare that there is no conflict of interests regarding the publication of this paper.

\section{Acknowledgments}

The authors gratefully acknowledge the financial support of CONACYT through Project 296395, CONACYT/Innovate UK through ACTin Project 268003 (México)/102729 (UK), and CONACYT/EU 7th CuVito Project 127151 (México)/ 263963 (EU). Noemi Jardón-Maximino wishes to thank CONACYT for the Scholarship no. 263275. Finally, the authors would like to thank V. Comparán-Padilla, G. Méndez-Padilla, and Myriam Lozano Estrada for their valuable technical support.

\section{References}

[1] E. Butovsky, I. Perelshtein, and A. Gedanken, "Air stable core-shell multilayer metallic nanoparticles synthesized by RAPET: fabrication, characterization and suggested application," Journal of Materials Chemistry, vol. 22, no. 30, p. $15025,2012$.

[2] A. Nowak, J. Szade, E. Talik, M. Zubko, D. Wasikowski, and M. Dulski, "Physicochemical and antibacterial characterization of ionocity $\mathrm{Ag} / \mathrm{Cu}$ powder nanoparticles," Materials Characterization, vol. 117, pp. 9-16, 2016.

[3] C. Ursino, R. Castro, E. Drioli, G. Lassaad, M. H. Albeirutty, and A. Figoli, "Progress of nanocomposite membranes for water treatment," Membranes, vol. 8, no. 2, p. 18, 2018.

[4] E. R. López, H. Ortega, G. Cadenas et al., "Foliar application of copper nanoparticles increases the fruit quality and the content of bioactive compounds in tomatoes," Applied Sciences, vol. 8, no. 7, article 1020, 2018.

[5] H. Hernández, S. González, A. Benavides, H. Ortega, G. Cadenas, and A. Juárez, "Effects of chitosan-PVA and Cu nanoparticles on the growth and antioxidant capacity of tomato under saline stress," Molecules, vol. 23, no. 1, p. 178, 2018.

[6] Z. Yu, C. Cui, B. Yang et al., "Size-controllable copper nanomaterials for flexible printed electronics," Journal of Materials Science, vol. 53, no. 18, pp. 12988-12995, 2018.

[7] N. K. Roy, C. S. Foong, and M. A. Cullinan, "Effect of size, morphology, and synthesis method on the thermal and sintering properties of copper nanoparticles for use in microscale additive manufacturing processes," Additive Manufacturing, vol. 21, pp. 17-29, 2018.

[8] N. Kaeffer, H. J. Liu, H. K. Lo, A. Fedorov, and C. Copére, “An $\mathrm{N}$-heterocyclic carbene ligand promotes highly selective alkyne semihydrogenation with copper nanoparticles supported on passivated silica," Chemical Science, vol. 9, no. 24, pp. 5366-5371, 2018.

[9] Y. Zhang, C. Ye, C. Guo, C. Gan, and X. Tong, " $\mathrm{In}_{2} \mathrm{O}_{3}$-modified $\mathrm{Cu} / \mathrm{SiO}_{2}$ as an active and stable catalyst for the hydrogenation of methyl acetate to ethanol," Chinese Journal of Catalysis, vol. 39, no. 1, pp. 99-108, 2018.
[10] X. Ren, X. Ji, Y. Wei et al., "In situ electrochemical development of copper oxide nanocatalysts within a TCNQ nanowire array: a highly conductive electrocatalyst for the oxygen evolution reaction," Chemical Communications, vol. 54, no. 12, pp. 1425-1428, 2018.

[11] Z. Wang, H. Du, Z. Liu, H. Wang, A. M. Asiri, and X. Sun, "Interface engineering of a $\mathrm{CeO}_{2}-\mathrm{Cu}_{3} \mathrm{P}$ nanoarray for efficient alkaline hydrogen evolution," Nanoscale, vol. 10, no. 5, pp. 2213-2217, 2018.

[12] R. Sierra, M. Pérez, G. Cadenas et al., "Synthesis of Copper Nanoparticles Coated with Nitrogen Ligands," Journal of Nanomaterials, vol. 2014, Article ID 361791, 8 pages, 2014.

[13] R. Betancourt, P. Reyes, and B. Puente, "Synthesis of copper nanoparticles by thermal decomposition and their antimicrobial properties," Journal of Nanomaterials, vol. 2014, Article ID 980545, 5 pages, 2014.

[14] R. Sierra, M. Pérez, G. Cadenas et al., "Synthesis of copper nanoparticles using mixture of allylamine and polyallylamine," Journal of Nanomaterials, vol. 2015, Article ID 367341, 9 pages, 2015.

[15] N. Dhas, C. Raj, and A. Gedanken, "Synthesis, characterization, and properties of metallic copper Nanoparticles," Chemistry of Materials, vol. 10, no. 5, pp. 1446-1452, 1998.

[16] A. Ponce and K. Klabunde, "Chemical and catalytic activity of copper nanoparticles prepared via metal vapor synthesis," Journal of Molecular Catalysis A: Chemical, vol. 225, no. 1, pp. 1-6, 2005.

[17] R. Zhou, X. Wu, X. Hao, F. Zhou, H. Li, and W. Rao, "Influences of surfactants on the preparation of copper nanoparticles by electron beam irradiation," Nuclear Instruments and Methods in Physics Research Section B: Beam Interactions with Materials and Atoms, vol. 266, no. 4, pp. 599-603, 2008.

[18] H. Zhu, C. Zhang, and Y. Yin, "Rapid synthesis of copper nanoparticles by sodium hypophosphite reduction in ethylene glycol under microwave irradiation," Journal of Crystal Growth, vol. 270, no. 3-4, pp. 722-728, 2004.

[19] I. Lisiecki and M. Pileni, "Synthesis of copper metallic clusters using reverse micelles as microreactors," Journal of the American Chemical Society, vol. 115, no. 10, pp. 3887-3896, 1993.

[20] R. Tilaki, A. Iraji, and S. Mahdavi, "Size, composition and optical properties of copper nanoparticles prepared by laser ablation in liquids," Applied Physics A, vol. 88, no. 2, pp. 415-419, 2007.

[21] L. Quoc, J. Hwa, and C. Woo, "Copper nanoparticles incorporated with conducting polymer: efects of copper concentration and surfactants on the stability and conductivity," Journal of Colloid and Interface Science, vol. 365, no. 1, pp. 103-109, 2012.

[22] B. Park, S. Jeong, D. Kim, J. Moon, S. Lim, and J. Kim, "Synthesis and size control of monodisperse copper nanoparticles by polyol method," Journal of Colloid and Interface Science, vol. 311, no. 2, pp. 417-424, 2007.

[23] J. Yang, S. Yang, and T. Okamoto, "Synthesis of copper monolayer and particles at aqueous-organic interface," Surface Science, vol. 600, pp. 318-320, 2006.

[24] Y. Wang and T. Asefa, "Poly(allylamine)-stabilized colloidal copper nanoparticles: synthesis, morphology, and their surface-enhanced raman scattering properties," Langmuir, vol. 26, no. 10, pp. 7469-7474, 2010. 
[25] S. Qiu, J. Dong, and J. Chen, "Preparation of $\mathrm{Cu}$ nanoparticles from water-in-oil microemulsions," Journal of Colloid and Interface Science, vol. 216, no. 2, pp. 230-234, 1999.

[26] J. Li and C. Liu, "Carbon-coated copper nanoparticles: synthesis, characterization and optical properties," New Journal of Chemistry, vol. 33, no. 7, pp. 1474-1477, 2009.

[27] N. Luechinger, E. Athanassiou, and W. Stark, "Graphenestabilized copper nanoparticles as an air-stable substitute for silver and gold in low-cost ink-jet printable electronics," Nanotechnology, vol. 19, no. 44, article 445201, 2008.

[28] F. Effenberger, M. Sulca, M. Machini, R. Couto, P. Kiyohara, and G. Machado, "Copper nanoparticles synthesized by thermal decomposition in liquid phase: the influence of capping ligands on the synthesis and bactericidal activity," Journal of Nanoparticle Research, vol. 16, no. 11, article 2588, 2014.

[29] J. Langford and A. Wilson, "Scherrer after sixty years: a survey and some new results in the determination of crystallite size," Journal of Applied Crystallography, vol. 11, no. 2, pp. 102-113, 1978.

[30] A. Monshi, "Modified Scherrer equation to estimate more accurately nano-crystallite size using XRD," World Journal of Nano Science and Engineering, vol. 2, no. 3, pp. 154-160, 2012.

[31] O. Mondal, A. Datta, D. Chakravorty, and M. Pal, "Ultrafine narrow dispersed copper nanoparticles synthesized by a facile chemical reduction method," MRS Communications, vol. 3, no. 2, pp. 91-95, 2013

[32] S. Saivova, S. Vorob'ev, R. Nokolaeva, and Y. Mikhlin, "Conditions for the formation of copper nanoparticles by reduction of copper(II) ions with hydrazine hydrate solutions," Russian Journal of General Chemistry, vol. 80, no. 6, pp. 1122-1127, 2010.

[33] Joint Committee on Powder Diffraction Standards, International Centre for Diffraction Data, Swarthmore, PA, USA, 1989.

[34] C. H. Caicedo, G. Ayala, A. C. Agudelo, and R. A. Vargas, "Effect of glycerol on the electrical properties, phase behavior and water vapor permeability in films based on potato starch," Revista Colombiana de Física, vol. 42, pp. 439-448, 2010.

[35] L. Dobrzanski and I. Czaja, "Effect of nanowires and nanoparticles of copper on the structure and properties of the nanocomposite polymeric materials," Archives of Materials Science and Engineering, vol. 75, pp. 18-29, 2015.

[36] J. Kim and Y. Chung, "Copper nanoparticle-catalyzed crosscoupling of alkyl halides with Grignard reagents," Chemical Communications, vol. 49, no. 94, pp. 11101-11103, 2013.

[37] O. Akhavan and E. Ghandi, " $\mathrm{Cu}$ and $\mathrm{CuO}$ nanoparticles immobilized by silica thin films as antibacterial materials and photocatalysts," Surface and Coating Technology, vol. 205, no. 1, pp. 219-223, 2010. 


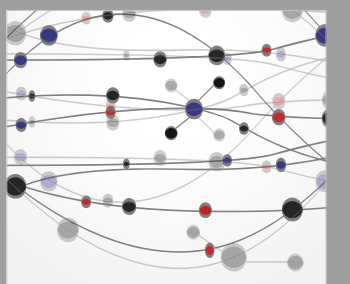

The Scientific World Journal
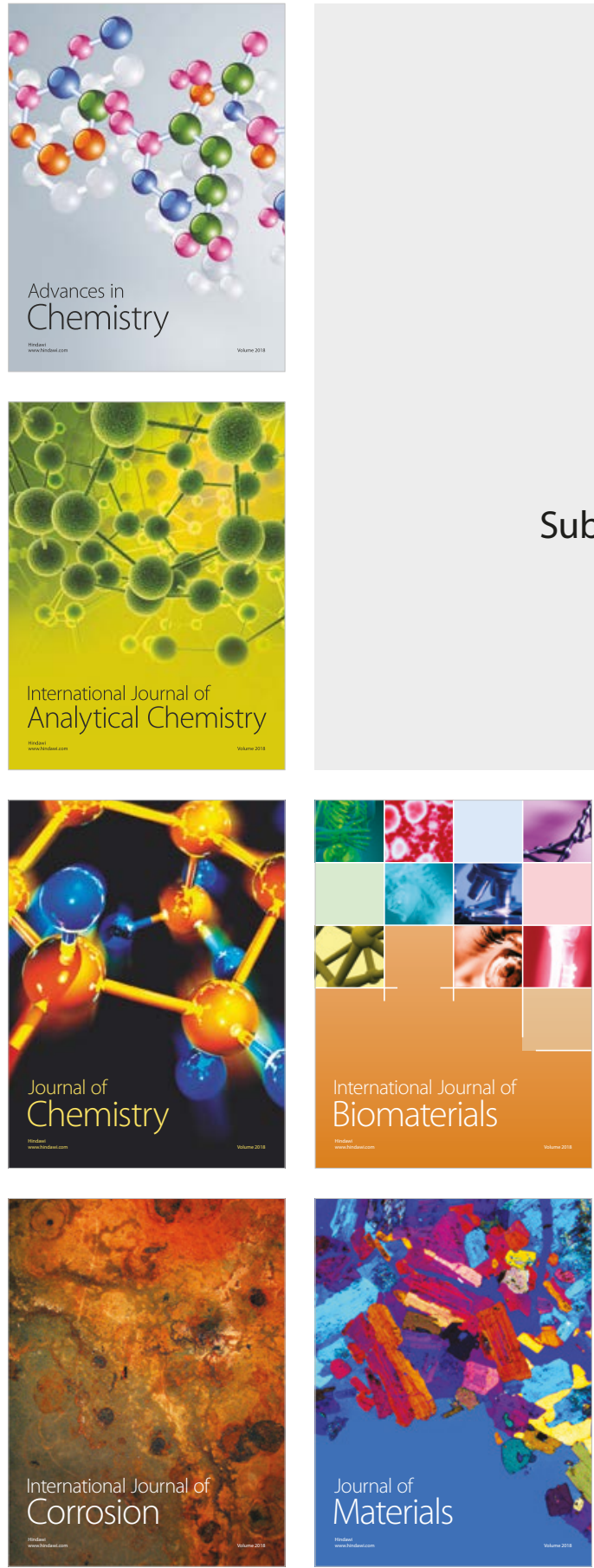

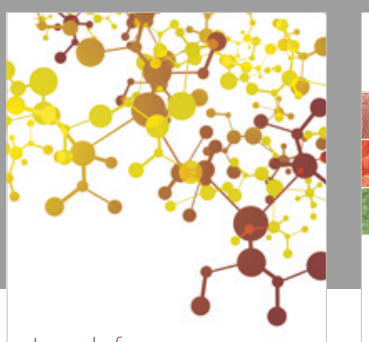

Journal of

Applied Chemistry
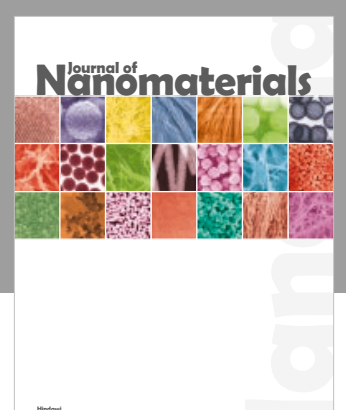

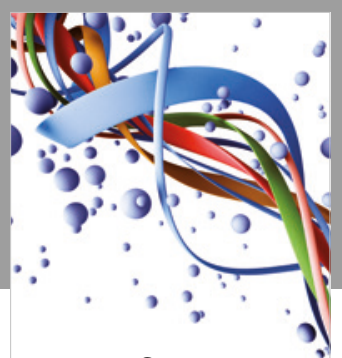

Scientifica

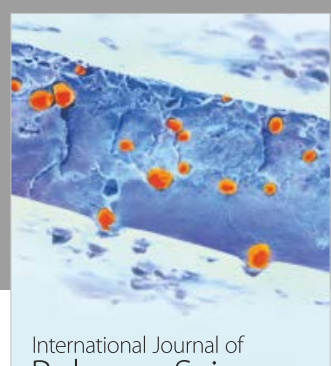

Polymer Science

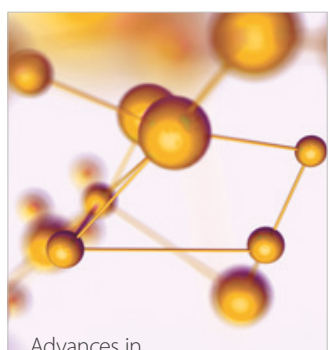

Physical Chemistry
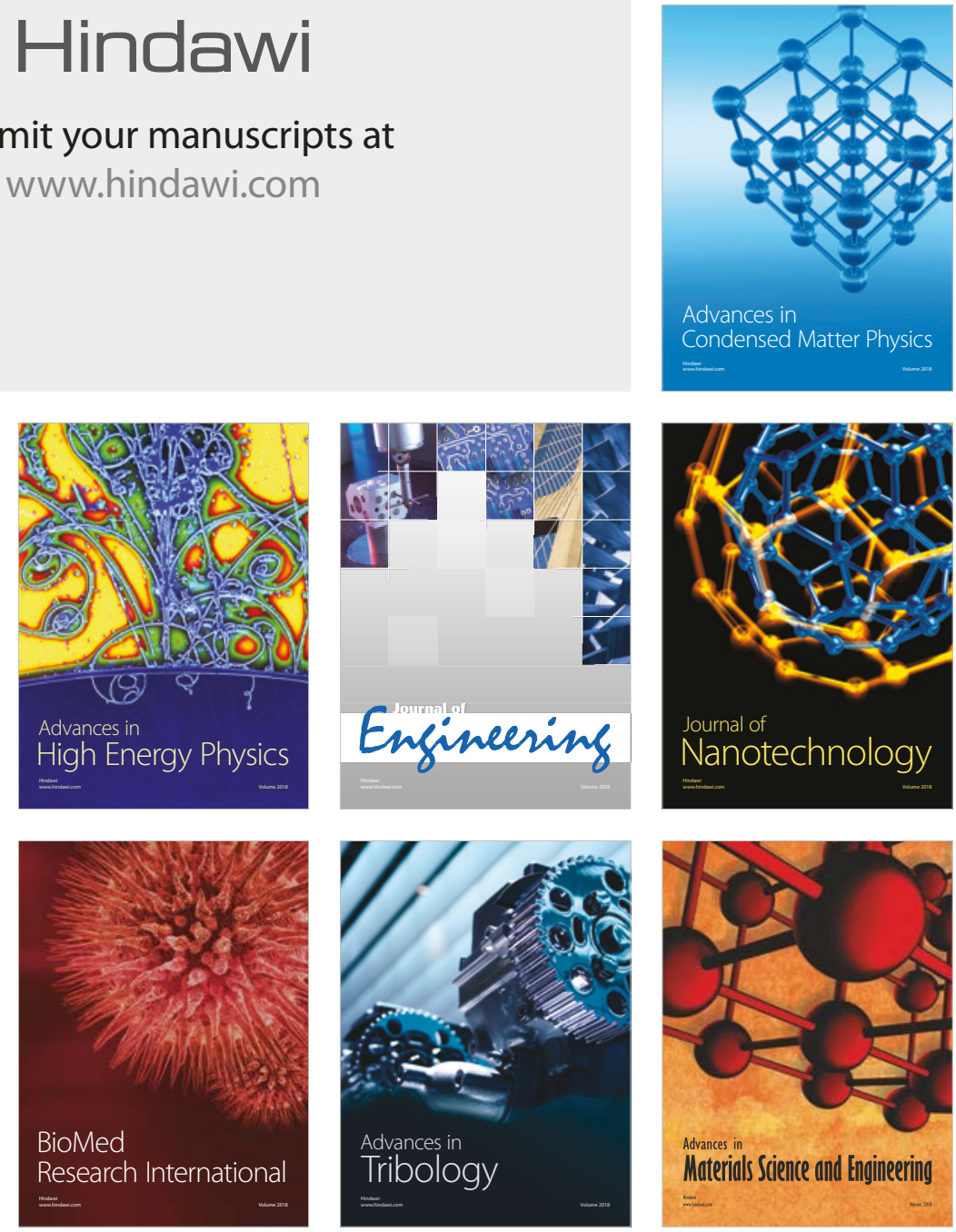